\section{Aplicação de uma metodologia de screening para avaliar a mortalidade por câncer em municípios selecionados do Estado de Minas Gerais, Brasil}

\author{
Screening methodology application to evaluate \\ cancer mortality in selected cities in the State \\ of Minas Gerais, Brazil
}

Ubirani B. Otero ${ }^{1}$

Berenice N. Antoniazzi 2

Lene H. S. Veiga 3

Silvana R. Turci 1

Gulnar Azevedo e Silva Mendonça ${ }^{1}$
${ }^{1}$ Coordenação de Prevenção e Vigilância, Instituto Nacional de Câncer. Rio de Janeiro, Brasil. 2 Programa de Avaliação e Vigilância, Secretaria de Estado de Saúde de Minas Gerais, Belo Horizonte, Brasil. ${ }^{3}$ Instituto de Radioproteção e Dosimetria, Comissão Nacional de Energia Nuclear, Rio de Janeiro, Brasil.

Correspondência U. B. Otero

Área de Vigilância do Cânce Ocupacional e Ambiental, Coordenação de Prevenção e Vigilância, Instituto Nacional de Câncer. Rua dos Inválidos 212, $2 \underline{a}$ andar, Rio de Janeiro, $R J$ 20231-048, Brasil. uotero@inca.gov.br

\begin{abstract}
An epidemiological investigation was launched in several cities in southern Minas Gerais State, Brazil, considering the possibility of increased cancer incidence due to high exposure to natural radiation. First, the cancer mortality patterns were assessed to determine whether there was an increase in cancer deaths and to discuss the possible risk factors related to such an increase. The study proposed the use of a screening methodology based on standardized mortality ratio (SMR) in order to classify priority areas for future studies. Cities considered high priority for further investigation were: Andradas, for lung cancer in men $(S M R=208$ (106-310)) and liver cancer in women (SMR = 403 (104-701)); Poços de Caldas, leukemia in men and women (SMR = 284 (156-412)) and SMR = 211 (111-312), respectively); Pouso Alegre, leukemia in men (SMR = 333 (127-540)) and hematological cancers in women $(S M R=257$ (188-396)). Epidemiological studies are necessary to evaluate the role of radiation and other risk factors in these cancers and thus to support future preventive and control measures.
\end{abstract}

Mortality Rate; Neoplasms; Background Radiation

\section{Introdução}

Atualmente o câncer representa a terceira mais importante causa de morte na população brasileira, após as doenças cardiovasculares e causas externas. No entanto, há uma marcante heterogeneidade da mortalidade por câncer entre as regiões geográficas brasileiras. Essas diferenças estão relacionadas principalmente ao desenvolvimento econômico, à dieta, ao tabagismo, a viroses e a exposições ambientais e ocupacionais, que, de alguma forma, estão ligados também às desigualdades sociais 1 .

A Secretaria de Estado de Saúde de Minas Gerais (SES-MG), o Instituto Nacional de Câncer (INCA) e o Instituto de Radioproteção e Dosimetria da Comissão Nacional de Energia Nuclear (IRD/CNEN) têm recebido várias solicitações de investigação de aumento do número de casos de câncer em municípios da região sul de Minas Gerais (Caldas, Poços de Caldas, Andradas, Muzambinho, Pouso Alegre, Poço Fundo, Ibitiúra de Minas e Araxá). No caso específico dos municípios do Planalto de Poços de Caldas e da cidade de Araxá, existe uma preocupação quanto a uma possível associação do aumento na incidência de câncer com a exposição à radiação natural, tendo em vista que a região do planalto é mundialmente reconhecida como uma Área de Radiação Natural Elevada 2,3.

No entanto, embora a radiatividade natural em algumas dessas regiões apresente valores 
mais elevados quando comparada com a de outras cidades do Brasil, essas maiores exposições não são representativas da exposição média de toda a população que vive nesses locais, uma vez que os níveis de radioatividade mais elevados, geralmente, restringem-se às pequenas áreas próximas às anomalias.

A classificação das áreas brasileiras de radioatividade natural elevada tem sido questionada, uma vez que critérios como homogeneidade da exposição e o número de pessoas realmente expostas não têm sido levados em consideração nesse tipo de classificação ${ }^{4}$.

Recentemente, Veiga et al. 5 realizaram um levantamento da exposição ao radônio e à radiação externa gama na população da área urbana e rural do Município de Poços de Caldas. Os autores reportam que, para a área urbana, foram encontrados valores médios de concentração de radônio dentro de casa da ordem de $60 \mathrm{Bqm}^{-3}$ e de taxa de dose gama externa na ordem de $0,16 \mu \mathrm{Gyh}-1$. Esses valores não caracterizam uma alta exposição e são compatíveis com os valores medidos em áreas de background normal de radiação como a cidade do Rio de Janeiro 6,7. Somente a área rural de Poços de Caldas, onde a maioria das anomalias geológicas ocorre, apresenta maiores valores de concentração de atividade de radônio e taxa de dose gama externa, $220 \mathrm{Bqm}^{-3}$ e $0,20 \mu \mathrm{Gyh}^{-1}$, respectivamente.

Levando em consideração somente essas duas exposições, que se constituem na maior contribuição para a dose total de radiação natural, os autores estimaram uma dose média de $1,6 \mathrm{mSv}$ ano-1 para a população que vive na área urbana de Poços de Caldas e 5,7mSv ano-1 para a população rural. A dose média mundial devido à exposição à radiação natural é de $2,4 \mathrm{mS}$ ano-1, na qual a exposição ao radônio e a exposição gama externa contribuem com cerca de $70 \%$ da dose total 8 .

Muitas vezes, o local reconhecido como área de radioatividade natural elevada tem causado receio à população, fazendo com que atribuam à radiação possíveis aumentos da incidência de câncer, assim como ocorrências de má-formação em crianças e outras doenças que não são necessariamente radio-induzidas.

Muitas áreas de alta radioatividade natural no mundo têm sido foco de vários estudos de dosimetria, estudos radiobiológicos e epidemiológicos. A elevada radioatividade natural nessas áreas é devida principalmente a estruturas geológica e geoquímica do solo ou à presença de águas termais radioativas nessas regiões. A radiação cósmica também pode contribuir significantemente em áreas de altas altitudes 9 .

Essas áreas estão localizadas na costa sudoeste da Índia 10, em Yangjiang, China 11, em
Ramsar e Mahallat no Irã 12, em Badgastein na Áustria 13, em Lake Miri, Sudão 14, nos Estados Unidos e Canadá 15 e no Brasil 4.

Os estudos epidemiológicos conduzidos nessas áreas ainda não foram capazes de demonstrar consistentemente qualquer efeito adverso à saúde associado à exposição à radiação ionizante 8,16 .

Existem várias dificuldades em se conduzir pesquisas sobre os efeitos na saúde para as baixas faixas de dose como as encontradas nas áreas de radioatividade natural elevada. Algumas dessas dificuldades são relacionadas à necessidade de condução de estudos analíticos (caso-controle e coorte), de um longo tempo de observação no caso de estudos de coorte, necessidade de um grande número de indivíduos envolvidos para se alcançar poder estatístico, de avaliação individual da dose para todos os indivíduos do estudo e da necessidade de controlar e distinguir os possíveis vieses e fatores de confundimentos, assim como a dificuldade de interpretação de dados que, muitas vezes, podem ser controversos 17 .

Uma vez que nenhuma dessas regiões possui um registro de câncer de base populacional, não se pode afirmar nem negar que exista uma maior incidência de casos de câncer na região quando comparada com outras regiões com condições sócio-econômicas similares. Veiga \& Koifman 18 avaliaram o padrão de mortalidade por câncer de algumas regiões brasileiras ditas como sendo de radioatividade natural elevada, como Poços de Caldas, Araxá e Guarapari, utilizando a razão de mortalidade padronizada (RMP) por câncer, tendo a população dos respectivos estados como população padrão. Os resultados mostram que, para Poços de Caldas e Guarapari, a mortalidade por câncer é maior do que se poderia esperar, tendo como base a população de referência. Para Poços de Caldas, observou-se um aumento significativo na mortalidade por câncer de estômago, pulmão, mama e leucemia. Para Guarapari, um aumento significativo na mortalidade foi observado para câncer de esôfago, estômago, pulmão e próstata, enquanto que, para Araxá, nenhum excesso de óbito por câncer foi observado.

Os autores ressaltam que esses resultados são importantes, uma vez que se desconhecia, até então, o padrão de mortalidade por câncer dessas regiões. Com esses dados, não se pode negar, por exemplo, que exista um aumento na mortalidade por câncer em Poços de Caldas. No entanto, deve-se levar em consideração, na interpretação desses resultados, não só os vários fatores de risco associados ao câncer, assim também como os importantes vieses relacionados aos dados de mortalidade. 
Tendo em vista o aumento da demanda de investigações a respeito de possíveis aumentos de câncer no Estado de Minas Gerais, o INCA, juntamente com a SES-MG e o IRD/CNEN, iniciou um projeto de investigação do padrão de mortalidade por câncer de alguns municípios selecionados de Minas Gerais, uma vez que nenhum desses municípios possui registros de base populacional.

Reconhecendo os vieses relacionados aos dados de mortalidade, o objetivo deste artigo é propor uma metodologia de screening, baseada nos dados de mortalidade por câncer, para priorizar quais tipos de câncer e quais municípios devem ser avaliados em futuras investigações.

\section{Metodologia}

Dados de mortalidade por câncer foram levantados para oito municípios do Estado de Minas Gerais (Poços de Caldas, Caldas, Andradas, Muzambinho, Pouso Alegre, Poço Fundo, Ibitiúra de Minas e Araxá). Os municípios incluídos neste estudo fazem parte do Planalto de Poços de Caldas e arredores e estão localizados na região sul do Estado de Minas Gerais, com exceção de Araxá que, embora não faça parte do Planalto de Poços de Caldas nem da macrorregião sul, foi incluído neste estudo devido à demanda por investigação epidemiológica e por ser também uma área reconhecida como de radioatividade natural elevada.

Os dados de mortalidade por câncer, no período de 1998 a 2002, obtidos do Sistema de Informações sobre Mortalidade (SIM) 19, foram avaliados segundo sexo e município de residência para os seguintes intervalos de idade: $0-9,10$ 19, 20-29, 30-39, 40-49, 50-59, 60-69, 70-79 e 80+. As localizações primárias de câncer avaliadas foram: todas as neoplasias malignas (C00-D09), estômago (C16), fígado (C22), pulmão (C33-C34), mama (C50), próstata (C61), bexiga (C67), leucemias (C91-95) e os cânceres hematológicos (linfomas e mielomas) (C82-C90).

A RMP é dada por: número de mortes observadas/número de mortes esperadas (x 100\%). A RMP foi calculada para cada município e cada macrorregião, tendo o Estado de Minas Gerais como população de referência. O número de óbitos esperados foi estimado multiplicando-se a taxa de mortalidade específica da população de referência segundo sexo, faixa etária e período ao número de pessoas por sexo e faixa etária dos municípios selecionados. Dados relativos à população no ano 2000 (meio do período) foram obtidos pelo Instituto Brasileiro de Geografia e Estatística.
Com o objetivo de identificar quais localizações primárias de câncer e municípios deveriam ser priorizados em futuras investigações, adotouse uma abordagem de screening com os seguintes critérios:

- Baixa prioridade: localizações primárias de câncer com valores de RMP abaixo de $100 \%$, estatisticamente significantes ou não (IC95\%);

- Média prioridade: localizações primárias de câncer com valores de RMP acima de 100\%, não significantes estatisticamente (IC95\%);

- Alta prioridade: localizações primárias de câncer com valores de RMP acima de 100\%, significantes estatisticamente (IC95\%);

- Altíssima prioridade: localizações primárias de câncer com valores de RMP acima de $200 \%$, estatisticamente significativos (IC95\%).

\section{Resultados}

A Tabela 1 apresenta a população dos municípios avaliados neste estudo, assim como o respectivo Índice de Desenvolvimento Humano Municipal (IDHM), que compreende a média dos índices de desenvolvimento municipal de renda, longevidade e educação para o ano de 2000.

O IDHM não varia muito entre os municípios selecionados, indicando que as condições socioeconômicas desses municípios são semelhantes. O menor IDHM é do Município de Ibitiúra de Minas (0,775), e o maior valor é do Município de Poços de Caldas $(0,841)$. No entanto, quanto à população residente, há uma grande diferença entre os municípios selecionados. Municípios com populações pequenas terão um menor número de óbitos observados, o que dificulta a análise e interpretação das RMP.

Tabela 1

População e Índice de Desenvolvimento Humano Municipal (IDHM) dos municípios selecionados neste estudo, 2000.

\begin{tabular}{lcc}
\hline Município & População & IDHM \\
\hline Andradas & 32.968 & 0,812 \\
Araxá & 78.997 & 0,799 \\
Caldas & 12.766 & 0,782 \\
Ibitiúra de Minas & 3.301 & 0,775 \\
Muzambinho & 20.589 & 0,801 \\
Poço Fundo & 15.148 & 0,774 \\
Poços de Caldas & 135.627 & 0,841 \\
Pouso Alegre & 106.776 & 0,826 \\
\hline
\end{tabular}


A Tabela 2 apresenta, para ambos os sexos, os municípios e localizações primárias de câncer que foram considerados como de baixa prioridade em futuras investigações, tendo em vista apresentarem valores de RMP menores que 100\%. Em Araxá, Caldas, Muzambinho e Poço Fundo, não houve excesso de óbitos, em homens, para câncer de estômago: RMP (IC95\%) = 90 (39-142); 96 (39-142); 88 (0-188); 79 (0-187), respectivamente, e, em Araxá e Poços de Caldas, também em homens, não houve excesso para câncer de fígado RMP = 70 (0-149) e 72 (14-130), respectivamente. Nota-se que, dentre os municípios selecionados, somente em Araxá, sexo masculino, para câncer de pulmão, observou-se uma RMP < 100\% (RMP = 37 (8-67)). Estudos sobre câncer de estômago, fígado e mama não devem ser encarados como prioritários em Andradas e Araxá (sexo feminino).

Quando consideramos todas as causas de óbitos por neoplasias, os municípios de Araxá e Poço Fundo devem ser considerados de baixa prioridade em futuras investigações para ambos os sexos, apresentando valores de RMP menores que $100 \%: \mathrm{RMP}=92(77-108)$ e RMP $=77(48-$ 106), respectivamente.

As Tabelas 3 e 4 apresentam os municípios e localizações primárias de câncer, para os sexos masculino e feminino, respectivamente, que foram considerados como de média prioridade em futuras investigações, tendo em vista apresentarem valores de RMP maiores que $100 \%$, não estatisticamente significantes. Nessa categoria, encontra-se a maioria das localizações primárias avaliadas. Destaque para leucemia (sexo masculino), que apresentou valores aumentados em Andradas: RMP = 215 (0-513), Araxá: $\mathrm{RMP}=380$ (76-684), Caldas: RMP = 1709 (343384) e Muzambinho: RMPR = 145 (0-429). Para o sexo feminino, observou-se comportamento semelhante em relação à leucemia nos seguintes municípios: Andradas: RMP = 346 (0-826), Araxá: RMP = 157 (0-335), Muzambinho: RMP= 330 (0980), Poço Fundo: RMP = 820 (0-1955) e Pouso Alegre: RMP = 183 (69-295).

A Tabela 5 apresenta, para ambos os sexos, os municípios e localizações primárias de câncer que foram considerados como de alta prioridade em futuras investigações com valores de RMP entre $100 \%$ e $200 \%$, estatisticamente significantes.

Com exceção do câncer de pulmão [RMP = 147 (106-188)] e mama [RMP = 168 (122-213)] em Poços de Caldas, os outros municípios definidos como de alta prioridade em futuras investigações foram os que apresentaram RMP significativas para o conjunto de óbitos por todas as neoplasias.

A Tabela 6 apresenta, para ambos os sexos, os municípios e localizações que foram considerados como de altíssima prioridade em futuras investigações com valores de RMP maiores que $200 \%$, estatisticamente significantes.

Os municípios de Andradas, Poços de Caldas e Pouso Alegre apresentaram RMP significativas para todas as neoplasias malignas (Tabela 5). Esse excesso de óbitos para todas as neoplasias

Tabela 2

Municípios e localizações primárias que apresentaram valores de razão de mortalidade padronizada (RMP) menores que $100 \%$ e considerados como de baixa prioridade em futuras investigações.

\begin{tabular}{|c|c|c|c|c|c|c|}
\hline \multirow[t]{2}{*}{ Municípios } & \multicolumn{3}{|c|}{ Masculino } & \multicolumn{3}{|c|}{ Feminino } \\
\hline & Causa de óbito * & Observado & RMP (IC95\%) & Causa de óbito * & Observado & RMP (IC95\%) \\
\hline Andradas & Estômago & 2 & $71(0-171)$ & Mama & 6 & $93(19-167)$ \\
\hline \multirow[t]{4}{*}{ Araxá } & Todas as neoplasias & 131 & $92(77-108)$ & Todas as neoplasias & 126 & $99(82-117)$ \\
\hline & Pulmão & 6 & $37(8-67)$ & Fígado & 2 & $62(0-149)$ \\
\hline & Fígado & 3 & $70(0-149)$ & Estômago & 4 & $43(1-85)$ \\
\hline & Estômago & 12 & $90(39-142)$ & Mama & 13 & $78(36-121)$ \\
\hline Caldas & Estômago & 3 & $96(0-205)$ & Mama & 1 & $90(0-266)$ \\
\hline \multirow[t]{2}{*}{ Muzambinho } & Estômago & 3 & $88(0-188)$ & Mama & 2 & $71(0-169)$ \\
\hline & Bexiga & 2 & $94(0-224)$ & - & - & - \\
\hline \multirow[t]{2}{*}{ Poço Fundo } & Todas as neoplasias & 27 & $77(48-106)$ & Todas as neoplasias & 17 & $76(40-112)$ \\
\hline & Estômago & 2 & $79(0-187)$ & Mama & 1 & $44(0-130)$ \\
\hline Poços de Caldas & Fígado & 6 & $72(14-130)$ & Fígado & 9 & $98(34-163)$ \\
\hline Pouso Alegre & - & - & - & Fígado & 5 & $88(11-165)$ \\
\hline
\end{tabular}

* Classificação Internacional de Doenças - 10a Revisão. 
Municípios e localizações primárias de câncer para o sexo masculino considerados de média prioridade em futuras investigações por apresentarem valores de razão de mortalidade padronizada (RMP) maiores que $100 \%$,

não estatisticamente significantes.

\begin{tabular}{|c|c|c|c|}
\hline Municípios & Causa de óbito * & Observado & RMP (IC95\%) \\
\hline \multirow[t]{5}{*}{ Andradas } & Leucemia & 2 & $215(0-513)$ \\
\hline & Hematológicos & 3 & $152(0-324)$ \\
\hline & Fígado & 1 & $181(0-534)$ \\
\hline & Estômago & 16 & $188(96-280)$ \\
\hline & Bexiga & 2 & $435(0-1.038)$ \\
\hline \multirow[t]{4}{*}{ Araxá } & Leucemia & 6 & $380(76-684)$ \\
\hline & Hematológicos & 5 & $103(13-193)$ \\
\hline & Bexiga & 3 & $126(0-270)$ \\
\hline & Estômago & 12 & $90(39-142)$ \\
\hline \multirow[t]{5}{*}{ Caldas } & Todas as neoplasias & 35 & $107(71-142)$ \\
\hline & Pulmão & 5 & $125(15-234)$ \\
\hline & Leucemia & 4 & 1.709 (34-3.384) \\
\hline & Hematológicos & 2 & $226(0-538)$ \\
\hline & Fígado & 1 & $225(0-666)$ \\
\hline Ibitiúra de Minas & Todas as neoplasias & 8 & $102(31-172)$ \\
\hline \multirow[t]{3}{*}{ Muzambinho } & Todas as neoplasias & 44 & $100(70-129)$ \\
\hline & Pulmão & 7 & $153(40-268)$ \\
\hline & Leucemia & 1 & $145(0-429)$ \\
\hline \multirow[t]{2}{*}{ Poço Fundo } & Pulmão & 4 & $110(2-219)$ \\
\hline & Fígado & 2 & $233(0-556)$ \\
\hline \multirow[t]{3}{*}{ Poços de Caldas } & Estômago & 40 & $127(87-166)$ \\
\hline & Bexiga & 10 & $102(39-164)$ \\
\hline & Hematológicos & 15 & $184(91-277)$ \\
\hline Pouso Alegre & Hematológicos & 12 & 188 (82-295) \\
\hline
\end{tabular}

* Classificação Internacional de Doenças - 10ạ Revisão.

deve estar sendo influenciado por algumas localizações primárias com valores de RMP acima de $200 \%$, estatisticamente significativos (Tabela 6). É o caso de Andradas, que apresentou uma RMP elevada para câncer de fígado: RMP $=403$ (104701) e Pouso Alegre, para leucemia: RMP $=333$ (127-540), ambos no sexo masculino. Destaque deve ser dado para Poços de Caldas que apresentou RMPs acima de $200 \%$, para leucemia, em ambos os sexos: $\mathrm{RMP}=284(156-412)$ em homens e RMP = 211 (111-312) em mulheres.

\section{Discussão}

A utilização de uma metodologia de screening para identificar os municípios e localizações primárias de câncer prioritários em futuros estudos se mostrou bastante útil, tendo em vista que apontou somente quatro municípios (Poços de Caldas, Pouso Alegre, Andradas e Caldas) e qua- tro localizações primárias de câncer (leucemia, hematológicos e fígado). No entanto, há de se considerar que os resultados encontrados para populações pequenas, como a de Caldas e Andradas, devem ser vistos com cuidado, tendo em vista o pequeno número de óbitos observados (4 e 7, respectivamente).

Os resultados deste estudo não permitem apontar fatores de risco que justifiquem os excessos de óbitos observados. A demanda de investigação epidemiológica nos municípios foi motivada por uma possível associação com a exposição à radiação ionizante. No entanto, dentre os municípios analisados, somente Poços de Caldas, Pouso Alegre e Andradas apresentaram excesso de óbitos significativos. Sendo que Pouso Alegre não está localizado em uma área de radiatividade natural elevada. Enquanto o Município de Araxá, que é reconhecidamente uma área de radiatividade natural elevada, não apresentou excesso de óbitos. Os resultados de mortalidade por câncer 
Tabela 4

Municípios e localizações primárias para o sexo feminino considerados de média prioridade em futuras investigações por apresentarem valores de razão de mortalidade padronizada (RMP) maiores que 100\%, não estatisticamente significantes.

\begin{tabular}{|c|c|c|c|}
\hline Municípios & Causa de óbito * & Observado & RMP (IC95\%) \\
\hline \multirow[t]{5}{*}{ Andradas } & Todas as neoplasias & 69 & $125(96-159)$ \\
\hline & Pulmão & 9 & $247(86-409)$ \\
\hline & Leucemia & 2 & $346(0-826)$ \\
\hline & Hematológicos & 3 & $195(0-415)$ \\
\hline & Bexiga & 1 & $548(0-1621)$ \\
\hline \multirow[t]{3}{*}{ Araxá } & Pulmão & 10 & $138(53-224)$ \\
\hline & Leucemia & 3 & $157(0-335)$ \\
\hline & Hematológicos & 8 & $202(62-341)$ \\
\hline \multirow[t]{3}{*}{ Caldas } & Todas as neoplasias & 30 & $129(83-175)$ \\
\hline & Pulmão & 4 & $308(6-610)$ \\
\hline & Fígado & 1 & $697(0-2065)$ \\
\hline \multirow[t]{2}{*}{ Ibitiúra de Minas } & Todas as neoplasias & 8 & $181(55-306)$ \\
\hline & Mama & 1 & $690(0-2043)$ \\
\hline \multirow[t]{5}{*}{ Muzambinho } & Todas as neoplasias & 40 & $113(78-149)$ \\
\hline & Pulmão & 3 & $155(0-330)$ \\
\hline & Leucemia & 1 & $330(0-980)$ \\
\hline & Fígado & 3 & $323(0-689)$ \\
\hline & Bexiga & 1 & $754(0-2231)$ \\
\hline \multirow[t]{3}{*}{ Poço Fundo } & Leucemia & 2 & $820(0-1955)$ \\
\hline & Fígado & 2 & $394(0-940)$ \\
\hline & Estômago & 1 & $379(0-1123)$ \\
\hline \multirow[t]{4}{*}{ Poços de Caldas } & Pulmão & 20 & $119(67-170)$ \\
\hline & Hematológicos & 10 & $148(56-239)$ \\
\hline & Estômago & 20 & $117(65-168)$ \\
\hline & Bexiga & 5 & $330(41-619)$ \\
\hline \multirow[t]{4}{*}{ Pouso Alegre } & Pulmão & 19 & $178(98-258)$ \\
\hline & Leucemia & 10 & $182(69-295)$ \\
\hline & Estômago & 13 & $123(56-190)$ \\
\hline & Bexiga & 4 & $415(8-821)$ \\
\hline
\end{tabular}

* Classificação Internacional de Doenças - 10a Revisão.

Tabela 5

Municípios e localizações primárias considerados como de alta prioridade em futuras investigações apresentando valores de razão de mortalidade padronizada (RMP) entre 100\% e 200\%, estatisticamente significantes.

\begin{tabular}{|c|c|c|c|c|c|c|}
\hline \multirow[t]{2}{*}{ Municípios } & \multicolumn{3}{|c|}{ Masculino } & \multicolumn{3}{|c|}{ Feminino } \\
\hline & Causa de óbito * & Observado & RMP (IC95\%) & Causa de óbito * & Observado & RMP (IC95\%) \\
\hline Andradas & Todas as neoplasias & 92 & $128(102-155)$ & & & \\
\hline \multirow[t]{3}{*}{ Poços de Caldas } & Todas as neoplasias & 314 & $121(107-134)$ & Todas as neoplasias & 298 & $127(112-141)$ \\
\hline & Pulmão & 49 & 147 (106-188) & & & \\
\hline & Mama & 51 & $168(122-213)$ & & & \\
\hline Pouso Alegre & Todas as neoplasias & 283 & $157(138-175)$ & Todas as neoplasias & 232 & $149(130-168)$ \\
\hline
\end{tabular}

* Classificação Internacional de Doenças - 10a Revisão. 
Municípios e localizações primárias considerados como de altíssima prioridade em futuras investigações apresentando valores de razão de mortalidade padronizada (RMP) maiores que $200 \%$, estatisticamente significantes.

\begin{tabular}{|c|c|c|c|c|c|c|}
\hline \multirow[t]{2}{*}{ Municípios } & \multicolumn{3}{|c|}{ Masculino } & \multicolumn{3}{|c|}{ Feminino } \\
\hline & Causa de óbito * & Observado/Esperado & RMP (IC95\%) & Causa de óbito * & Observado/Esperado & RMP (IC95\%) \\
\hline Andradas & Pulmão & $16 / 8$ & 208 (106-310) & Fígado & $7 / 2$ & $403(104-701)$ \\
\hline Poços de Caldas & Leucemia & $19 / 7$ & $284(156-412)$ & Leucemia & $17 / 8$ & $211(111-312)$ \\
\hline Pouso Alegre & Leucemia & $10 / 3$ & $333(127-540)$ & Hematológicos & $13 / 5$ & $257(118-396)$ \\
\hline
\end{tabular}

* Classificação Internacional de Doenças - 10ạ Revisão.

para Araxá e Poços de Caldas são semelhantes aos reportados por Veiga \& Koifman 18.

Dois principais aspectos devem ser considerados quando avaliamos esses resultados. O primeiro diz respeito à qualidade das informações dos óbitos e o segundo aos possíveis fatores de risco associados a esses cânceres.

A qualidade das informações contidas nas declarações de óbito e sua utilização em estudos epidemiológicos têm sido analisadas por diversos pesquisadores, que destacam a maior confiabilidade da classificação da causa básica de morte nos casos de câncer 20,21.

A análise da mortalidade segundo causas pode apresentar deficiências relacionadas à cobertura do SIM (sub-registro e subenumeração), sendo também prejudicada por problemas inerentes à declaração da causa básica da morte por parte dos médicos e, às vezes, falhas na sua codificação, embora, do ponto de vista da qualidade, é possível verificar uma melhora gradativa dos dados de mortalidade 22 . Os municípios avaliados neste estudo apresentam uma proporção média de causas mal definidas de $5 \%$ a $18 \%$ em relação aos óbitos totais no período de 1998 a 2002, que é compatível com a proporção média de causas mal definidas registrada para o Estado de Minas Gerais (14\%) para o mesmo período.

Dentre os maiores municípios analisados: Poços de Caldas, Pouso Alegre e Araxá, o último não apresentou valores de razão de mortalidade significativos para nenhuma localização primária, mesmo considerando que os problemas relativos a sub-registro, subenumeração, falhas na codificação sejam comuns aos três municípios.

Outra dificuldade na utilização de dados de mortalidade diz respeito ao local de residência reportado na declaração de óbito, uma vez que não se pode assegurar que a pessoa sempre tenha residido naquele município. Tendo em vista esses possíveis vieses, um estudo de validação dos óbitos está sendo executado pela SES-MG e
INCA com o objetivo de avaliar a qualidade das informações contidas nas declarações de óbitos.

O fato de alguns municípios apresentarem RMPs muito elevadas, porém com intervalos de confiança não significativos para algumas das localizações primárias de câncer (Araxá, Andradas, Ibitiúra de Minas, Muzambinho e Poço Fundo), pode ser explicado, em parte, pelo pequeno número de habitantes (menor que 100 mil) e conseqüentemente pequeno número de óbitos observados. As RMPs podem variar muito entre as áreas de uma mesma região, devido às variabilidades das taxas por estarem baseadas em pequenos números de eventos observados 23 .

Outro aspecto que deve ser considerado é quanto à população de referência utilizada. $\mathrm{O}$ Estado de Minas Gerais possui 853 municípios, sendo que os municípios da macrorregião sul e centro são os mais desenvolvidos. Uma vez que a maioria dos municípios selecionados neste estudo localiza-se na macrorregião sul, que pode ter taxas de mortalidade distintas das observadas no Estado de Minas Gerais como um todo, foram calculadas as RMPs utilizando a taxa de mortalidade por câncer da macrorregião sul como população de referência. Os resultados foram muito semelhantes ao deste estudo, apontando como alta e altíssima prioridade os mesmos municípios e localizações primárias que a análise com a população do estado como referência.

Otero et al. 24 reportam valores de RMP para as macrorregiões do Estado de Minas Gerais, utilizando o próprio estado como população de referência. Os maiores valores de RMP, para algumas localizações primárias, estatisticamente significativos, foram encontrados para a macrorregião centro, seguida da macrorregião sul e Triângulo do Norte. Comparando-se os valores das RMPs das macrorregiões com os valores das RMPs dos municípios selecionados neste estudo, observamos que, para leucemia, em Poços de Caldas e Pouso Alegre, as RMPs são maiores que 
o maior valor estatisticamente significativo da macrorregião sul para ambos os sexos: 126 (108144), sexo feminino e 120 (103-136), sexo masculino. Da mesma forma, as RMPs encontradas para câncer de pulmão, sexo feminino, em Poços de Caldas, são maiores que as reportadas para a macrorregião sul: 107 (98-115).

Dessa forma, os significantes valores de RMP para câncer de pulmão, leucemia e câncer de mama encontrados em Poços de Caldas e leucemia e cânceres hematológicos em Pouso Alegre sugerem que realmente existe um excesso de óbitos por esses cânceres nesses municípios que devem ser avaliados tendo em vista os possíveis fatores de risco ambientais associados à etiologia desses cânceres.

Estudos epidemiológicos em áreas de radioatividade natural elevada no mundo têm sido conduzidos há muito tempo, tendo em vista que as populações que vivem nessas áreas são uma importante fonte de informações sobre os efeitos da exposição crônica a baixas doses de radiação ionizante. No entanto, até o presente, não se conseguiu quantificar o risco dessa exposição, tendo obtido somente informações limitadas 8 .

As incertezas em estudos epidemiológicos envolvendo baixas doses podem ser atribuídas a diversos fatores: dosimetria inadequada, faixas de doses estreitas, grupos controles inadequados ou inapropriados e ainda os efeitos decorrentes dos fatores de confundimento que são potencialmente mais problemáticos quando a expectativa de incidência dos efeitos a serem detectados é pequena. Entretanto, o maior problema nesses tipos de estudos é a falta de poder estatístico, devido ao pequeno número de pessoas envolvidas e aos baixos valores de dose 25 .

Cardis 26 conclui que, com os estudos realizados até o presente, é difícil avaliar a existência e a possível magnitude do risco à saúde associado com residentes de áreas de radiação natural elevada. A autora recomenda que seja realizado um estudo multicêntrico envolvendo várias populações que vivem em áreas de radioatividade natural elevada em diversos países, tendo em vista que o uso de protocolos comuns de dosimetria e desenho epidemiológico permitiriam uma comparação direta dos resultados e uma análise combinada, o que maximizaria as informações desses estudos.

Uma análise dos principais fatores de risco relacionada às neoplasias apontadas como de alta prioridade neste estudo se faz necessária. Para câncer de pulmão, o fumo é o fator de risco preponderante e deve ser considerado em futuros estudos que visem explicar o excesso de óbitos encontrado nos municípios de Poços de Caldas e Andradas, em indivíduos do sexo mas- culino. Dados da literatura ressaltam que $90 \%$ das mortes por câncer de pulmão poderiam ser evitadas se o tabagismo fosse eliminado, bem como cerca de $30 \%$ das outras mortes por câncer. Entre os tipos de câncer relacionados ao uso do tabaco, incluem-se o de pulmão, boca, laringe, faringe, esôfago, estômago, fígado, pâncreas, bexiga, rim e colo de útero. Dessa forma, o tabagismo é um fator de risco preponderante e representa um problema de saúde pública, não somente nos países desenvolvidos, como também em países em desenvolvimento, como o Brasil 27,28,29. Outros fatores incluem o radônio, o amianto, o arsênico, a sílica e o cromo. Fatores da dieta como o alto consumo de gorduras e álcool também devem ser considerados, bem como a história familiar 30.

Os dados sobre a proporção de fumantes disponíveis para o Brasil são muito antigos: datam de 1989 quando foi realizada a Pesquisa Nacional de Saúde e Nutrição 31 . Havia, naquele ano, mais de 30 milhões de fumantes no país.

Dados do Inquérito Domiciliar sobre Comportamentos de Risco e Morbidade Referida de Doenças e Agravos Não Transmissíveis, realizado em 15 capitais e o Distrito Federal nos anos de 2002 e 2003 32, revelaram que a prevalência de tabagismo diminuiu, porém ainda é muito elevada. Na capital do Estado de Minas Gerais, Belo Horizonte, por exemplo, a prevalência foi de $26,1 \%$ $(23,0-29,1)$, sexo masculino, e $15,9 \%(13,8-18,0)$, sexo feminino. Em geral, os homens apresentaram maiores prevalências de tabagismo do que as mulheres em todas as cidades pesquisadas.

O segundo fator de risco para câncer de pulmão, depois do fumo, é a exposição ao radônio. $\mathrm{O}$ radônio é um gás radioativo, originado pelo decaimento radioativo do urânio e posteriormente do rádio, presente naturalmente na crosta terrestre. A importância do radônio, do ponto de vista da radioproteção, decorre da radiotoxicidade de seus produtos á-emissores de meia-vida curta, o Po218 e o Po214, e de sua maior abundância no meio ambiente 33 . A energia das partículas alfa, liberadas pelos produtos de decaimento, o Po218 e o Po214, depositadas nas células do epitélio respiratório, é considerada a causa do câncer de pulmão associado à exposição ao radônio.

Vários estudos em trabalhadores de minas subterrâneas, de urânio e outros minérios contendo urânio, que estão expostos a radônio e seus produtos de decaimento, têm demonstrado um aumento no risco de câncer de pulmão quando comparados com um grupo não exposto 14 . Embora a exposição ao radônio seja universalmente reconhecida como a segunda causa de câncer de pulmão, o risco desse tipo de câncer para o público em geral, devido principalmente 
à exposição dentro de casa, ainda era obtido pela extrapolação dos dados obtidos nos estudos em mineiros, uma vez que os estudos epidemiológicos de radônio em residências não foram capazes de consistentemente demonstrar esse risco. Somente recentemente obtiveram-se evidências diretas desse risco através da análise combinada de diversos estudos conduzidos na Europa, América do Norte e China 34,35,36.

Para leucemia, diversos fatores de risco foram reportados na literatura: exposição ocupacional ao benzeno, radiações ionizantes, óxido de etileno, os agentes antineoplásicos, os campos eletromagnéticos, os agrotóxicos clorados, o tabagismo e outros como a dieta 37 . Dentre os óbitos por leucemia em Poços de Caldas, observou-se igual distribuição dos óbitos por subtipos de leucemia (linfocítica, mielocítica e não específicas). Para Pouso Alegre, 50\% dos óbitos por leucemia foram pelo subtipo mielocítica. Quanto à faixa etária, a maioria dos óbitos ocorreu na população adulta ( $\geq 20$ anos), 94\% em Poços de Caldas e 70\% em Pouso Alegre.

Embora a leucemia seja um câncer reconhecidamente radio-induzido, poucas evidências existem acerca da sua associação com a exposição à radiação natural. Diversos estudos epidemiológicos visando estudar a associação entre leucemia e radiação natural foram realizados 38,39,40,41. Esses estudos não encontraram nenhuma associação significativa entre leucemia e radiação natural de background. Um estudo anterior na Escócia encontrou uma associação significativa entre leucemia e radiação natural. Mas, concluiu que, mesmo para a leucemia, uma interpretação apropriada das variações geográficas só pode ser feita quando os efeitos dos fatores sociais e econômicos também puderem ser levados em conta 42 . Knox et al. 43 reportaram uma associação estatisticamente significativa entre radiação natural de background e leucemia em crianças. No entanto, os resultados não foram confirmados numa publicação subseqüente 44 .

Em Poços de Caldas, ocorreu elevada RMP para o câncer de mama. Fatores como idade, his- tória familiar de câncer de mama, idade da menarca, do primeiro filho, da menopausa, obesidade, consumo de gordura elevado, dentre outros, podem ser citados como fatores associados ao desenvolvimento de câncer de mama 45 .

$O$ câncer de fígado, em Andradas, teve um alto valor de RMP significativo. No entanto, além do pequeno de número de óbitos observados, a informação de mortalidade por esse tipo de câncer deve ser analisada com restrições, por ser o fígado um local freqüente de metástases, nem sempre adequadamente informadas pelos laudos dos óbitos. Alguns fatores de risco para câncer de fígado podem ser apontados: histórico de cirrose hepática, a infestação por esquistossomose em áreas endêmicas, exposição a substâncias químicas como o cloreto de vinil, os arsenicais inorgânicos e o Thorotraste (solução de dióxido de tório) e as aflatoxinas (produzidas pelo fungo Aspergillus flavus em grãos e cereais) 46,47 .

Dessa forma, os futuros estudos epidemiológicos analíticos a serem conduzidos devem contemplar todos os possíveis fatores de risco associados a essas neoplasias consideradas como de alta prioridade.

\section{Conclusões}

A metodologia de screening proposta neste estudo se mostrou eficiente em identificar os municípios e localizações primárias de câncer que devem ser priorizados em futuros estudos que visem elucidar os fatores de risco ambientais e/ou ocupacionais relacionados aos excessos de óbitos encontrados. As futuras investigações devem incluir estudos observacionais analíticos que permitam estabelecer relação de nexo causal entre os fatores de risco ambientais e a incidência de câncer. Dessa forma, será possível subsidiar futuras medidas de prevenção e controle do câncer. Por fim, essa metodologia poderá ser utilizada para outras situações de demanda de investigação epidemiológica a fim de priorizar esforços e recursos. 


\section{Resumo}

Iniciou-se uma investigação epidemiológica em municípios do sul de Minas Gerais, Brasil, considerando a possibilidade de aumento na incidência de câncer devido a maior exposição à radiação natural em alguns deles. Buscou-se, preliminarmente, conhecer o padrão de mortalidade por câncer, avaliar se existe excesso de óbitos e discutir os possíveis fatores de risco associados a esse aumento. Propõe-se uma metodologia de screening com base na razão de mortalidade padronizada (RMP) para classificar os municípios prioritários em estudos futuros. Os municípios considerados de altíssima prioridade para investigação foram: Andradas - câncer de pulmão em homens [RMP = 208 (106-310)] e figado em mulheres [RMP = 403 (104-701)]; Poços de Caldas - leucemia em homens e mulheres $(R M P=284$ (156-412) e RMP = 211 (111-312), respectivamente); Pouso Alegre - leucemia em homens [RMP = 333 (127540)] e cânceres hematológicos em mulheres [RMP = 257 (188-396)]. Estudos epidemiológicos devem ser conduzidos para avaliar a contribuição da radiação e outros fatores de riscos relacionados ao câncer e assim subsidiar futuras medidas de prevenção e controle.

Coeficiente de Mortalidade; Neoplasias; Radiação de Fundo

\section{Colaboradores}

U. B. Otero, B. N. Antoniazzi e L. H. S. Veiga efetuaram os cálculos, discutiram os resultados e os analisaram junto com a equipe técnica. Após essa etapa, redigiram o artigo, tendo trabalhado nele até a sua etapa final. S. R. Turci e G. A. S. Mendonça contribuíram nas reuniões de discussão, análise dos dados e revisão final.

\section{Agradecimentos}

Aos pesquisadores Marcelo Moreno dos Reis, Fátima Sueli Neto Ribeiro, Cláudio Noronha, Marise Souto Rebelo (Coordenação de Prevenção e Vigilância/INCA), Cristiana Ferreira Jardim de Miranda, Jandira Maciel, Thays Aparecida Leão d'Alessandro, Renato Azeredo Teixeira e (SES-MG); Moacyr Cipriani (CNEN) e Marcos Eduardo de Andrade (Santa Casa de Misericórdia, Poços de Caldas); Horst Fernandes (IRD/CNEN) e Juliana Villarde (Coordenação Geral de Vigilância em Saúde Ambiental/Ministério da Saúde) pela participação nas discussões e colaborações diversas. Agradecemos também o apoio administrativo dos técnicos Bruno Mariano, Fátima Regina Souza (Coordenação de Prevenção e Vigilância/INCA) e Ângela Maria do Amparo (SES-MG).

\section{Referências}

1. Wünsch Filho V, Moncau JE. Mortalidade por câncer no Brasil 1980-1995: padrões regionais e tendências temporais. Rev Assoc Med Bras 2002; 48:250-7.

2. Penna-Franca E, Ribero CC, Teitakowski M, Londres H, Santos PL, Albuquerque HA. Survey of radioactive content of food grown on Brazilian areas of high natural radioactivity. Health Phys 1965; 11:1471-84.

3. Penna-Franca E, Almeida JC, Becker J, Emmerich M, Roser FX, Kegel G, et al. Status of investigations in the brazilian areas of high natural radioactivity. Health Phys 1965; 11:699-712.

4. Veiga LHS, Sachet I, Melo V, Amaral ECS. Brazilian areas of high background radiation - are they really high? In: Proceedings of the $5^{\text {th }}$ International Conference on High Levels of Natural Radiation and Radon Areas: Radiation Dose and Health Effects. Munich: Federal Office for Radiation Protection/Institute of Radiation Hygiene; 2000. p. 63-5.

5. Veiga LHS, Koifman, S, Melo V, Sachet I, Amaral ECS. Estimating indoor radon risk at a Brazilian area of high natural radiation. J Environ Radioact 2002; 70:161-76.
6. Sachet I. Caracterização da radiação gama ambiental em áreas urbanas utilizando uma unidade móvel de rastreamento [Tese de Doutorado]. Rio de Janeiro: Instituto de Biociências Nucleares, Universidade do Estado do Rio de Janeiro; 2002.

7. Magalhães MH, Amaral ECS, Sachet I, Rochedo ER. Radon-222 in Brazil: an outline of indoor and outdoor measurements. J Environ Radioact 2003; 67:131-43.

8. United Nations Scientific Committee on the Effects of Atomic Radiation. Sources and effects of ionizing radiation. Report to the General Assembly, with scientific annexes. New York: United Nations; 2000.

9. United Nations Scientific Committee on the Effects of Atomic Radiation. Sources and effects of ionizing radiation. Report to the General Assembly, with scientific annexes. New York: United Nations; 1993. 
10. Sunta CM. A review of the studies of the high background areas of the S-W Coast of India. In: Proceedings of International Conference on High Level Natural Radiation Areas. Vienna: International Atomic Energy Agency; 1990. p. 3-7. (IAEA Publication Series, 71).

11. Wei L, Zha Y, Tao Z. Epidemiological investigation of radiological effects in high background radiation areas of Yiangjiang, China. J Radiat Res 1990; 31:119-36.

12. Sohrabi MM, Beitollahi MM, Lasemi Y, Amin SE. Origin of a new high level natural radiation area in hot spring region of Mahallat, Central Iran. In: Proceedings of the $4^{\text {th }}$ International Conference on High Levels of Natural Radiation. Vienna: International Atomic Energy Agency; 1996. p. 3-7.

13. Pohl-Rüling J, Steinhäusler F, Pohl E. Radiation exposure and resulting risk due to residence and employment in a radon spa. In: Vohra KG, Mishra UC, Pillai KC, Sadasivan S, editors. Proceedings of the $2^{\text {nd }}$ Special Symposium on Natural Radiation Environment. Bombay: Wiley Eastern Ltd.; 1982. p. 107.

14. Mukhtar OM, Elkhangi RFA. Environmental study of radionuclide in Miri Lake area, Nuba Mountains. In: International Conference on High Level Natural Radiation Areas, Book of Abstracts. Vienna: International Atomic Energy Agency; 1990. p. 15.

15. National Council on Radiation Protection. Exposure of the population in the United States and Canada from natural background radiation. Bethesda: National Council on Radiation Protection; 1992.

16. Yoshinaga S, Tokonami S, Akiba S. Residential radon and childhood leukemia a meta-analysis of published studies. In: Proceedings of the $6^{\text {th }}$ International Conference on High Levels of Natural Radiation and Radon Areas. Osaka: Kinki University; 2005. p. 430-1. (International Congress Series, 1276).

17. Wei L. High background radiation area - an important source of exploring the health effects of low dose ionizing radiation. In: Proceedings of the $4^{\text {th }}$ International Conference on High Levels of Natural Radiation: Radiation Doses and Health Effects. Tokyo: Elsevier; 1997. p. 1-13.

18. Veiga LHS, Koifman S. Pattern of cancer mortality in some Brazilian HBRAs. In: Proceedings of the $6^{\text {th }}$ International Conference on High Levels of Natural Radiation and Radon Areas. Osaka: Kinki University; 2005. p. 110-3. (International Congress Series, 1276).

19. Departamento de Informática do SUS. Sistema de informação sobre mortalidade. Dados de declaração de óbito 1997-2001. Brasília: Ministério da Saúde; 2004.

20. Monteiro GTR, Koifman RJ, Koifman S. Confiabilidade e validade dos atestados de óbito por neoplasias. II. Validação do câncer de estômago como causa básica dos atestados de óbito no Município do Rio de Janeiro. Cad Saúde Pública 1997; 13 Suppl 1:52-65.

21. Percy C, Staneck E, Gloecker L. Accuracy of cancer deaths certificates and its effects on cancer mortality statistics. Am J Public Health 1981; 71:24250.
22. Jorge MHPM, Gotlieb SLD, Laurenti R. A saúde no Brasil: análise do período de 1996 a 1999. Brasília: Organização Pan-Americana da Saúde; 2001.

23. Vacchino MN. Poisson regression in mapping cancer mortality. Environ Res 1999; 81:1-17.

24. Otero UB, Antoniazzi BN, Veiga LHS, Turci SR, Reis MM, Ribeiro FSN, et al. Avaliação do padrão de mortalidade por câncer em municípios selecionados e macrorregiões do Estado de Minas Gerais, entre 1998 e 2002. In: Anais do 2º Simpósio Nacional de Geografia e Saúde [CD-ROM]. Rio de Janeiro: Fundação Oswaldo Cruz; 2005.

25. United Nations Scientific Commitee on the Effects of Atomic Radiation. Sources and effects of ionizing radiation. Report to the General Assembly, with scientific annex. New York: United Nations; 1994.

26. Cardis E. Commentary on information that can be drawn from studies of areas with high levels of natural radiation. In: Proceedings of the $6^{\text {th }}$ International Conference on High Levels of Natural Radiation and Radon Areas. Osaka: Kinki University; 2005. p. 118-23. (International Congress Series, 1276).

27. Ministério da Saúde/Organização Pan-Americana da Saúde. Tabaco e pobreza, um círculo vicioso. Brasília: Ministério da Saúde/Organização PanAmericana da Saúde; 2004.

28. Cipriani M. Percepção de riscos e de impactos socioeconômicos da radioatividade [Tese de Doutorado]. Belo Horizonte: Pontifícia Universidade Católica de Minas Gerais; 2003.

29. International Agency for Research on Cancer. Tobacco smoking. IARC Monogr Eval Carcinog Risk Chem Hum 1986; 38:35-394.

30. Centers for Disease Controle e Prevention. Lung cancer: risk factors. http://www.cdc.gov/cancer/ lung/basic_info/risk_factors.htm (acessado em 21/Nov/2006).

31. Instituto Nacional de Alimentação e Nutrição. Pesquisa nacional sobre saúde e nutrição. Brasília: Instituto Nacional de Alimentação e Nutrição; 1990.

32. Instituto Nacional de Câncer. Inquérito domiciliar sobre comportamentos de risco e morbidade referida de doenças e agravos não transmissíveis, Brasil, 15 Capitais e Distrito Federal - 2002-2003. Rio de Janeiro: Instituto Nacional de Câncer; 2004.

33. Committee on the Biological Effects of Ionizing Radiations, National Research Council. Health risks of radon and other internally deposited alpha-emitters: BEIR IV. Washington DC: National Academies Press; 1988.

34. Lubin JH, Wang ZY, Boice JD, Blot WJ, Wang LD, Kleinerman RA. Risk of lung cancer and residential radon in China: pooled results of two studies. Int J Cancer 2004; 109:132-7.

35. Krewski D, Lubin JH, Zielinski JM, Alavanja M, Catalan VS, Field RW, et al. Residential radon and risk of lung cancer: a combined analysis of 7 North American case-control studies. Epidemiology 2005; 16:137-45. 
36. Darby S, Hill D, Auvinen A, Barros-Dios JM, Baysson H, Bochicchio F, et al. Radon in homes and risk of lung cancer: collaborative analysis of individual data from 13 European case-control studies. BMJ 2005; 330:223.

37. Ministério da Saúde/Organização Pan-Americana da Saúde. Doenças relacionadas ao trabalho. Manual de procedimentos para os serviços de saúde. Brasília: Ministério da Saúde; 2001.

38. Iwasaki T, Minowa M, Hashimoto S, Hayashi N, Murata M. Cancer mortality rates by geographic distribution and levels of natural radiation dose in Japan. In: Proceedings of the International Conference on High Level Natural Radiation Areas. Vienna: International Atomic Energy Agency; 1993. p. 503-14.

39. Tirmarche M, Rannou A, Mollie A. Epidemiology study of regional cancer mortality in France and natural radiation. Radiat Prot Dosimetry 1988; 24:479-82.

40. Edling C, Comba P, Axelson. Effects of low dose radiation - a correlation study. Scand J Work Environ Health DATA; 8 Suppl 1:59-64.
41. High Background Radiation Research Group. Health surveys in high background radiation areas in China. Science 1980; 209:877-80.

42. Court-Brown WM, Doll R, Spiers FW. Geographical variations in leukemia mortality in relation to background radiation and other factors. BMJ 1960; 1:1753-9.

43. Knox EG, Stewart AM, Gilman C. Background radiation and childhood cancer. J Radiol Prot 1988; 8:9-18.

44. Muirhead CR, Butland BK, Green BMR. Childhood leukemia and natural radiation. Lancet 1991; 337:503-4.

45. Greenwald P, Kramer BS, Weed DL. Cancer prevention and control. New York: Marcel Dekker; 1995.

46. Antony PP. Precursor lesions for liver cancer in humans. Cancer Res 1976; 36:2579-83.

47. Lutwick LI. Relation between aflatoxins and hepatitis B virus and hepatocellular carcinoma. Lancet 1979; 1:755.

Recebido em 04/Mai/2006

Versão final reapresentada em 13/Dez/2006

Aprovado em 12/Jan/2007 